\title{
Prevalence and Associated Factors of Injuries Related to Running: A Study among Runners in Sri Lanka
}

\author{
Mayooran $\mathrm{S}^{1, *}$, Nanayakkara S.D.I ${ }^{2}$, Rajaratne A.A.J ${ }^{2}$, Kotakadeniya H.M.S.R.B ${ }^{2}$ \\ ${ }^{1}$ Department of Physiotherapy, Faculty of Allied Health Sciences, University of Peradeniya, Sri Lanka \\ ${ }^{2}$ Faculty of Medicine, University of Peradeniya, Sri Lanka \\ *Corresponding author: ursmayoo22@gmail.com
}

Received January 06, 2019; Revised February 10, 2019; Accepted April 01, 2019

\begin{abstract}
Running is one of the popular sports worldwide and it is the sport of choice for many in Sri Lanka because of its convenience, health benefits and economical nature. However, the potential for running injuries also has been well documented in the world literature. But unfortunately, at present there is no database available on running injuries among Sri Lankan runners. Aim of this study is to generate scientific details on running injuries among runners in Sri Lanka. A cross sectional study was performed among 256 runners (133 short distance runners and 123 long distance runners) in Sri Lanka who perform at provincial and national level competitions. The prevalence of any form of injury was 50.39\% (129 injured athletes among 256 participants), while a total of 143 injuries sustained among 256 runners with the prevalence rate of $55.85 \%$. The prevalence of any form of injury among short distance runners was $46.61 \%$ and among long distance runners was $54.47 \%$. The body region showing the highest injury prevalence was the Knee joint (22.38\%) in both short and long distance runners. Hamstring (18.18\%), lower back (13.99\%), ankle (13.29\%) and calf (11.19\%) were the other common sites of injuries. Abnormal Q angle, flat foot and history of previous injuries were found as associated intrinsic risk factors, while not engaging in stretching exercises by holding a stretch $>20$ seconds, training at hard intensity and sudden alterations in training pattern were found as associated extrinsic risk factors for injuries in runners. Age, body mass index, leg length discrepancy, training time per week and running shoes were found to have no association with the occurrence of injuries in runners. The findings of this study revealed that injury rate is high and suggests that effective preventive strategies are needed to reduce the injury rate in runners in Sri Lanka.
\end{abstract}

\section{Keywords: running injuries, injury prevalence, Sri Lankan runners}

Cite This Article: Mayooran S, Nanayakkara S.D.I, Rajaratne A.A.J, and Kotakadeniya H.M.S.R.B, "Prevalence and Associated Factors of Injuries Related to Running: A Study among Runners in Sri Lanka." American Journal of Sports Science and Medicine, vol. 7, no. 2 (2019): 28-33. doi: 10.12691/ajssm-7-2-1.

\section{Introduction}

Running is one of the popular sports and it is the most popular physical activity enjoyed by people around the world. The running events vary in distance from short sprints to long distance races as well as races involving hurdles. Sprint races cover relatively short distances, such as the $100 \mathrm{~m}, 200 \mathrm{~m}$ and $400 \mathrm{~m}$ dashes. Long distance races cover such distances as $3000 \mathrm{~m}, 5000 \mathrm{~m}, 10,000 \mathrm{~m}$ and marathons. Running is the sport of choice for many and the popular physical activity, not only because of its health advantages but also because of its convenience and economical nature. However, the potential for running injuries such as knee injuries, hamstring injuries, ankle injuries and many other running related injuries also has been well documented in the world literature [1]. Training and competing in running events involves long periods of repetitive stress on the musculoskeletal system. As a result, the majority of running injuries are attributable to overuse of the musculoskeletal system [2].
At present there is no database or statistics with common running injuries, risk factors of injuries and rehabilitation of the injuries of Sri Lankan runners. Identifying the injury patterns and risk factors of injuries is essential to develop effective injury prevention strategies to minimize the injury rate and also to develop effective treatment protocols for the injuries. Physical structure, physical qualities, environmental factors and training methodologies in Sri Lankan athletes, are unique to our conditions. Therefore, conducting a study to investigate the injuries patterns among runners in Sri Lanka, and identifying the association of possible predisposing factors of the injuries seems to have an immense value and need. Thus, this study aimed to generate scientific details about injury prevalence, injury patterns and potential risk factors of injuries related to running among runners in Sri Lanka.

\section{Methodology}

A descriptive cross sectional study was performed among 256 runners in Sri Lanka who perform at provincial and 
national level competitions. Among them 133 were short distance runners and 123 were long distance runners. The study was carried out at the Exercise and Sport Sciences Laboratory, Faculty of Medicine, University of Peradeniya, Sri Lanka. The prevalence of injury was studied to identify the common injuries among Sri Lankan runners. Injuries were studied according to the anatomical body regions such as upper back, lower back, hip, hamstring, anterior thigh, knee, calf, shin, ankle, foot and injuries to other body regions. Running injury was defined as any reported muscle, soft tissue, joint or bone problem / injury resulting from running during practice or competition and requiring the runner to be removed from the practice or competition. Further, possible risk factors associated with injuries related to running were also studied in this study. Risk factors were divided into two groups as intrinsic factors and extrinsic factors. Age, BMI, history of previous injury and biomechanical factors - Q angle, leg length difference and flat foot were studied as intrinsic factors. Training related factors such as training time, type of training and history of running shoes were studied as extrinsic factors.

An interviewer administered questionnaire was used to obtain demographic data, history of previous injuries and training related information. Anthropometric and biomechanical measurements were taken by using standard methods. Height and weight were measured by using stadiometer and calibrated digital weighing scale respectively. Goniometer was used to measure $\mathrm{Q}$ angle. Goniometer was placed over the centre of the patella by positioning the bottom arm in line with the patella tendon and tibial tuberosity while upper arm was pointed to the anterior superior iliac spine (ASIS) of the ilium. Leg length measurement was taken from ASIS to medial malleolus of the leg. If the difference is more than $1.5 \mathrm{~cm}$ between sides, it was considered as leg length discrepancy. Normalized navicular height truncated measurement was used to identify the flat arched foot.

All collected data were entered and analyzed with statistical measures using SPSS (Vn.20. Prevalence of injury and injury pattern were explained as percentages of different types of injuries according to anatomical regions. Chi-square test and binominal logistic regression model were used to identify the relationship between the injuries and associated risk factors.

\section{Results}

\subsection{Prevalence of Injuries}

A total of 129 runners, 62 short distance runners and 67 long distance runners sustained injuries related to running among the study group. The prevalence of any form of injury in the total sample was 50.39\%. The prevalence of any form of injury among short distance runners was $46.61 \%$ and among long distance runners was $54.47 \%$. Of those 129 injured runners, 14 reported more than one injury. Accordingly a total of 143 injuries sustained among 256 runners with the prevalence rate of $55.85 \%$. The body region showing the highest injury prevalence was the Knee joint in both short and long distance runners. Hamstring, lower back, ankle and calf were the other common sites of injuries in both short and long distance runners.

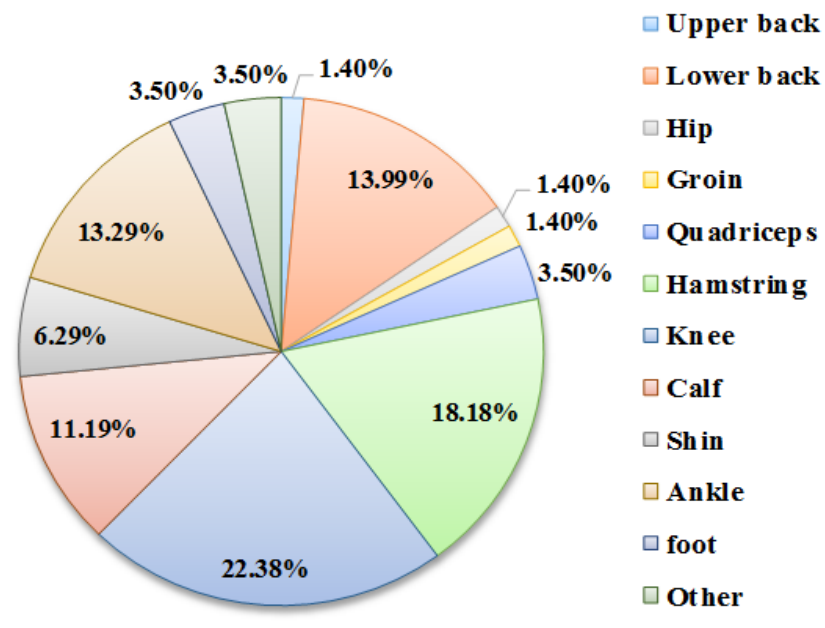

Figure 1. Injury pattern according to the site of the injury

Of the group who was injured, $79 \%$ of injuries were reported to have occurred during training while only $16 \%$ occurred during competitions. It was also reported that $62 \%$ of injuries were new injuries while $38 \%$ were recurrent injuries. Hamstring, lower back and calf injuries were reported as common sites for recurrent injuries among the study group.

\subsection{Factors Associated with Injuries}

\subsubsection{Intrinsic Factors}

\section{a. Age}

Athletes were categorized into four different age groups to study the association between age and occurrence of injuries. Categories for age were as follows; $\leq 20$ years, 21 - 25 years, $26-29$ years and 30 years and above. Minimum age was 18 and maximum was 32 in the study group. There was no significant association found between the age and occurrence of injuries in the total sample. But there was a significant association $(\mathrm{p}=0.002)$ found between the age and occurrence of injuries among long distance runners.

\section{b. BMI}

Majority of the runners in the study sample (75.78\%) had normal BMI, while $08.20 \%$ were in the underweight group, $16.02 \%$ were in the overweight group. There was no statistical significance association found between the BMI and occurrence of injuries in the study sample. However there was a trend noticed with BMI and occurrence of injuries with marginal significance $(p=0.056)$ among long distance runners indicated that long distance runners with higher BMI had higher tendency to get injured.

\section{c. Biomechanical factors: $\mathbf{Q}$ angle, Leg length difference and Foot arch}

89 runners (34.76\%) in the study group were found with abnormal Q angle. Of those, 56 (62.92\%) runners had increased Q angle and 60\% were reported with injuries. There was a statistical significant association found between $\mathrm{Q}$ angle and the occurrence of injuries among runners. 
Runners who had abnormal Q angle were more likely to get injured. There was no statistical significant association found between leg length difference and occurrence of injuries. But it was noticed that the percentage of injured athletes was high among runners who had leg length difference more than $1.5 \mathrm{~cm} .42$ runners in the study group were found with flat foot. Of those, 14 (33.33\%) runners had bilateral flat foot. There was a statistical significant association found between flat foot and occurrence of injuries. Runners who had flat foot were found to have a higher tendency to get injured.

Table 1. Association between biomechanical factors and running injuries

\begin{tabular}{lc|c|c}
\hline Variable & Category & Injury (\%) & Sig. (P value) \\
\hline \multirow{2}{*}{ Q angle } & Normal & $45.50 \%$ & \multirow{2}{*}{$0.032 *$} \\
& Abnormal & $59.55 \%$ & \\
\hline \multirow{2}{*}{ Leg length } & Yes $->1.5 \mathrm{~cm}$ & $63.63 \%$ & \multirow{2}{*}{0.076} \\
discrepancy & No - $<1.5 \mathrm{~cm}$ & $49.14 \%$ & \\
\multirow{2}{*}{ Flat foot } & Yes & $73.81 \%$ & \multirow{2}{*}{$0.001 *$} \\
& No & $45.79 \%$ & \\
\hline
\end{tabular}

* Significant association at $\mathrm{p}<0.05$.

When biomechanical factors were further analyzed to find out the association with site of injuries, significant association was found between abnormal Q-angle and the occurrence of Hamstrings injuries among short distance runners $(\mathrm{p}=0.009$, Binominal logistic regression test). Runners with abnormal Q angle showed 4.132 times greater odds of having hamstring injuries than runners with normal Q angle.

\section{d. History of previous injuries}

$41.8 \%$ of the athletes in the study group reported any form of previous injuries during within one year before the study period. The majority of those who had previous injuries (61.68\%) sustained with injuries during the study period. Of those, $57.57 \%$ had recurrent injuries at the same site of injury. There was a significant association found between the history of previous injuries and occurrence of injuries in runners in the study group $(p=0.001)$.

\subsubsection{Extrinsic Factors}

\section{a. Training Time}

The average number of hours trained in a week was studied to find out the association between training time and the occurrence of injuries. Training time was categorized into three groups as follows; < $10 \mathrm{hrs}$./week, $10-15$ hrs./week and $>15$ hrs./week. Majority of runners had their training for 10 - 15 hours in a week. Although the percentages of injured runners was slightly high among runners who trained more than 15 hours in a week, there was no significant association found between the hours of training and the occurrence of injuries.

b. Training habits: Warm-up, Stretching \& Cool down

The majority of the study sample (>95\%) reported that they do warm up exercises at least for 20 minutes before training and cool down exercises at least for 10 minutes after training. $81.64 \%$ reported that they do specific stretching exercises for major muscle groups by doing static stretching exercises before training. Of those, $69.37 \%$ of runners held a stretch for 15 - 20 seconds, whereas $30.63 \%$ held a stretch for > 20 seconds. Remaining $18.35 \%$ reported that they did not engage in specific stretching exercises other than warm-up exercises. There was a significant association found $(p=0.001)$ between doing specific stretching exercises and occurrence of injuries. Higher percentage (59.6 \%) of runners who did not engage in specific stretching exercises had injuries when compare to runners who engaged in specific stretching exercises. Engaging in stretching exercises by holding a stretch $>20$ seonds reduced the risk of injuries.

\section{c. Training intensity}

Training intensity was evaluated as degree of difficulty of training according to the athletes' perception. Training intensity was categorized in to three groups as 'Easy', 'Moderate' and 'Hard'. Among 256 athletes 110 reported it as being easy, 82 reported it as moderate and 64 reported it as hard. It was found that runners who underwent for 'hard' training according to their perception had a higher tendency $(71.87 \%)$ to get injured than runners who underwent for 'moderate' (54.87\%) and 'easy' (34.50\%) training.

\section{d. Training pattern and changes in the training}

$58.8 \%$ of the study sample reported doing alternative high and low intensity training on different days, $26.4 \%$ reported doing similar training every day and others reported different training on different days with other forms of training such as hill training, interval training and burst running.

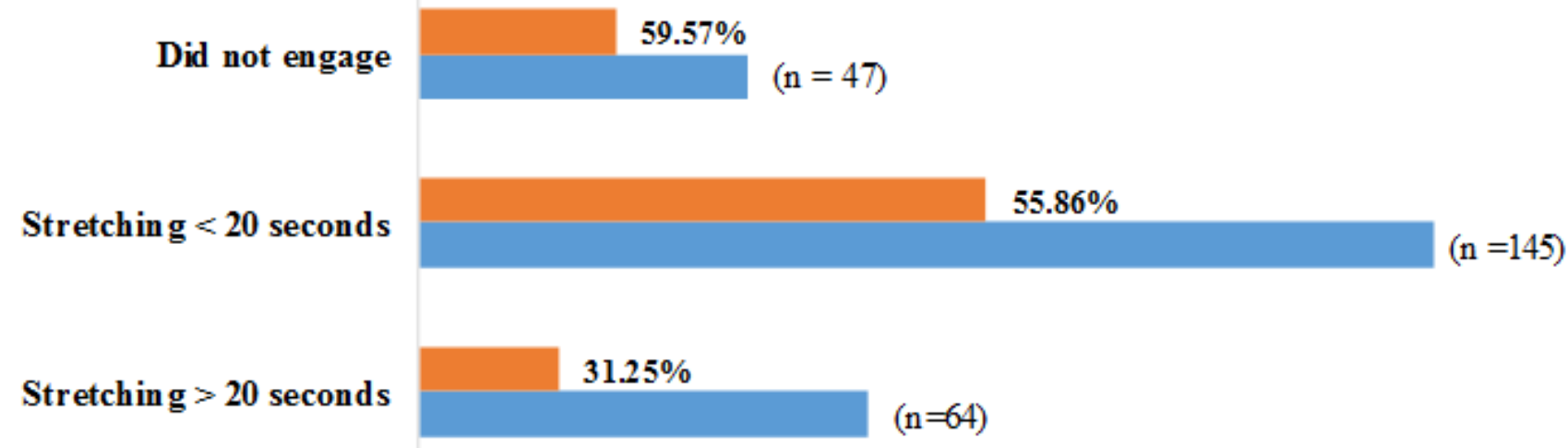

" Percentage of runn ers injured $\quad$ Numb er of runners engaged

Figure 2. Injuries among runners who engage in specific stretching exercises 
It was noticed that there was a strong association between sudden alteration in training pattern and onset of injuries. $88.0 \%$ of the injured athletes had changed their training pattern by increasing the distance, increasing the speed, hill running or by changing the training surface before the occurrence of the injury (Figure 3).
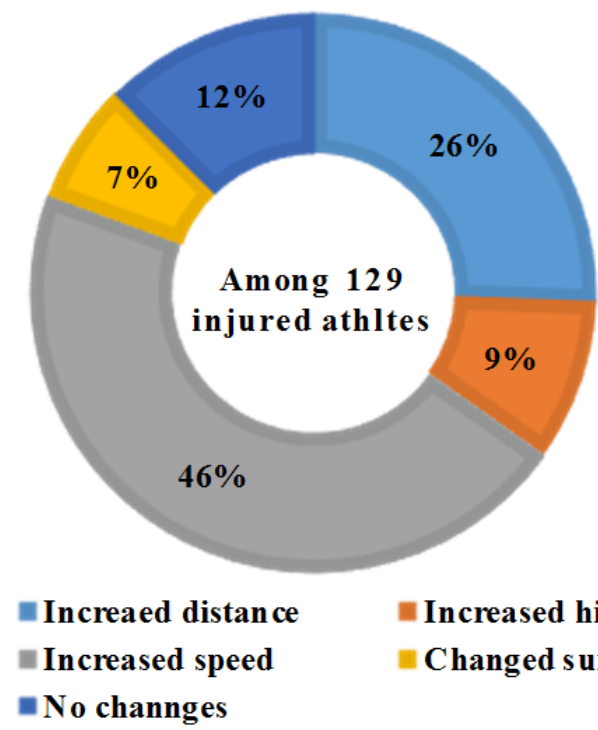

\section{Increased hill running "Changed surfaces}

Figure 3. Sudden alterations in training pattern in the injured athletes

\section{e. Type and usage of running shoes}

All the runners in the study group reported using standard running shoes during the training and competitions. It was found that $52.7 \%$ of runners in the study group ran in a pair of running shoes for approximately 06 - 12 months, whereas $40.7 \%$ of runners tended to use a pair of running shoes for a period of 12 - 24 months. Only a small percentage of runners (6.6\%) used a pair of shoes for less than 06 months. The mean duration of the usage of a pair of shoes of injured group was $12.1 \pm 6.9$ months and of non-injured group was $9.4 \pm 4.3$ months. There was no statistical difference found in the average duration of the usage of a pair of shoes between injured and non-injured group in the study group.

\section{Discussion}

The prevalence of injured athletes in this study was 50.39\% (129 injured athletes among 256 participants), while a total of 143 injuries sustained among 256 runners with the prevalence rate of $55.85 \%$. This is comparable with prevalence rates reported in previous studies done in other countries $[3,4]$. The findings of this study related to common sites of injuries among runners in Sri Lanka are also in agreement with previously published studies in other countries. According to the present study, the most common location of injuries was the Knee followed by hamstring, lower back, ankle and calf. A large number of literature states that the most common running injury occurs in the Knee [5,6]. Repetitive stress on the Knee joint during running, sudden twisting and bending activities can stress the knee joint and lead to injuries. The impact forces at the Knee joint resulting from the running can be two to three times body weight [7]. This can contribute to increase the occurrence of Knee injuries, particularly overuse injuries.
There is an inconsistency in the evidences available in the literature to determine whether age is significantly associated with the injuries in runners. The greater age was found to be a significant risk factor for incurring running injuries in previous studies because of the structural changes in the bone and soft tissues $[6,8,9]$. However, some researchers stated that older age is not a predictor for injury in runners and injury is less likely to occur in very young and older runners $[3,10]$. The results of the current study found that there was no statistical significant association between the BMI and occurrence of injuries in the study sample. This finding is comparable with previous studies [11,12]. Conflicting evidence was found in a study which reported, increased BMI (greater than $26 \mathrm{~kg} / \mathrm{m}^{2}$ ) was a protective factor against injury in men [6]. If the higher BMI due to the greater muscle mass, which may increase the muscle strength and thereby can reduce the risk of injures.

In the current study, there was a significant association found between $\mathrm{Q}$ angle and the occurrence of injuries among runners. Rauh et al. (2006) found that runners with an increased Q-angle $\left(>15^{\circ}\right)$ had a higher risk of lower limb injuries [13]. This could be due to a larger lateral pull on the patella against the lateral femoral condyle possibly contributing to patella malalignment and patellofemoral pain disorders [14]. Furthermore, Puckree et al., (2007) found that increased Q-angle $\left(>22^{\circ}\right)$ in Indian male runners were directly proportional to the incidence of knee injuries in runners [5]. The current study also found that there was a significant association between abnormal Q-angle and the occurrence of knee injuries. There is evidence that flat arched foot is significantly associated with the risk of injuries in runners. In this study, it was found that runners who had flat foot had a significant higher tendency to get injured. Changes in the foot arch may change the biomechanics and force distribution during running, thereby can increase the risk of injuries. A systematic review with meta-analysis with 27 studies reported a significant association between nonneutral foot types and lower extremity injuries and it was found that high arch and flat-foot foot types are associated with lower extremity injuries [15].

History of previous injury was found to be one of the risk factor strongly associated with the occurrence of running injuries in previous studies $[6,16,17]$. The current study also found the similar findings. It was found that the majority of those who had previous injuries sustained with recurrent injuries during the study period. Of those, $57.57 \%$ had recurrent injuries at the same site of injury. This possibly indicates the inadequate rehabilitation or early return to sport that could lead to the recurrence of injuries.

It was found that engaging in regular stretching and held a stretch for more than 20 seconds significantly reduced the risk of injury (31.25\%) than held a stretch for less than 20 seconds (55.86\%) and not engaging in specific stretching exercises (59.57\%). Lack of flexibility has been found as one of the major risk factor for sports injuries in many studies and the evidences shows that inadequate stretching increases the risk of running injuries [18]. A randomized trial study on pre-exercise stretching for prevention of lower-limb injury also reported a similar finding that stretching for 05 times; holding a stretch for more than 30 seconds reduced the risk of injury [12]. 
In this study, the training intensity according to the athletes' perception was assessed to find the association with injuries. It was found that athletes who underwent for 'hard' training had a higher tendency (71.87\%) to get injured than 'moderate' (54.87\%) and 'easy' (34.50\%) training according to their perception. This is possibly due to overuse injuries. A systematic review of interventions to prevent lower limb soft tissue running injuries, done by Yeung et al. (2001) also reported that exposure to a high training load increases the risk of injury [18].

The results of this current study showed a strong association between sudden alteration in training pattern and the occurrence of injuries. Majority of the injured athlete reported a sudden change in their training pattern before the occurrence of the injury. Jacobs \& Berson, (1986) reported a comparable finding that one third of those injured described they had changed their training just prior to their injuries [19]. A similar finding was reported by Johnston \& Taunton (2003) who found that $60 \%$ of running injuries in their study are the results of increasing distance too quickly [20]. Karageanes (2004) also agrees with this statement as a sudden increase in speed or distance can be a precipitating factor for running injuries [21]. This is possibly due to that a sudden increase in running speed or duration or intensity can overwhelm the ability for adaptive change, tissue repair, and result in injury.

In this study, there was no association found between the average duration of running shoes and the onset of running injuries. There are still inconsistencies in the evidences found in the literature in determining the association of running shoes with running injuries. However, it was suggested that running shoes needs to be changed regularly as shoes that exceed $700 \mathrm{~km}$ mark could lose the ability to absorb shock optimally which could in turn result in injury [22]. It was also suggested that wearing incorrect shoes that have insufficient height, rigid soles, twists easily or that are worn out can easily result in running injuries [23].

The variations in the findings of this study compare to the findings of the previous studies could be due to differences in sample size, variations in definitions of injury and different study methodologies. This could also be due to the different training backgrounds and environment of the participants in Sri Lanka and due to faulty training techniques as it was reported in our study that most injuries occurred while training than the competition. However, the findings of the present study, with an injury prevalence rate of 50.3\% among runners in Sri Lanka indicates the necessity to take serious measures to implement effective injury prevention programmes for runners in Sri Lanka.

\section{Conclusion}

Results of this study revealed that injury prevalence is high $(>50 \%)$ among Sri Lankan runners. Most of the injuries affected the Knee, Hamstring, lower back and Ankle and occurred during training. Abnormal Q angle, flat foot and history of previous injuries were found as associated intrinsic risk factors for injuries in runners. In extrinsic factors, not engaging in specific stretching exercises by holding a stretch $>20$ seconds, training at hard intensity and sudden alterations in training pattern were found to be as associated extrinsic risk factors for injuries in runners. Age was found to be associated with the risk of injuries among long distance runners. BMI, leg length discrepancy, average training time per week and running shoes were found to have no association with the occurrence of injuries in runners.

\section{References}

[1] Jacobsson, J., Toomas, T., Kowalski, J., Sverker Nilsson, Joakim Ekberg, Renstrom, P. (2011). Prevalence of Musculoskeletal Injuries in Swedish Elite Track and Field Athletes: American Journal of Sports Medicine: 40: 163

[2] McKenzie, D.C., Clement, D.B., Taunton, J.E. (1985). Running shoes: Orthotics and injuries. Sports Medicine; (2): 334-347.

[3] Van Middelkoop, M., Kolkman, J., Van Ochten, J., BiermaZeinstra, S. M. A., \& Koes, B. (2008). Prevalence and incidence of lower extremity injuries in male marathon runners. Scandinavian Journal of Medicine and Science in Sports, 18(2), 140-144.

[4] McKean, K. A., Manson, N. A., \& Stanish, W. D. (2006). Musculoskeletal injury in the masters runners. Clinical Journal of Sport Medicine, 16(2), 149-154.

[5] Puckree, T., Govender, A., Govender, T., \& Naidoo, P. (2007). The quadriceps angle and the incidence of knee injury in Indian long-distance runners. South African Journal of Sports Medicine, 19(1), 10-12.

[6] Taunton, J. E., Ryan, M. B., Clement, D. B., McKenzie, D. C., Lloyd-Smith, D. R., \& Zumbo, B. D. (2003). A prospective study of running injuries: the Vancouver Sun Run "In Training” clinics. British Journal of Sports Medicine, 37, 239-244.

[7] Knutzen K, Hart, L. (1996): Epidemiology of Sports Injuries. Champaign, Human Kinetics, 357-386.

[8] Robertson, B., Nicholl, J. P., Williams, B. T., \& Rossi, A. (1983) Popular Marathons: Forecasting Casualties. British Medical Journal Clinical Research Edition, 286105215 (286).

[9] Wen, D. Y., Puffer, J. C., \& Schmalzried, T. P. (1998). Injuries in runners: A prospective study of alignment. Clinical Journal of Sport Medicine, 8(3), 187-194.

[10] Satterthwaite, P., Norton, R., Larmer, P., \& Robinson, E. (1999). Risk factors for injuries and other health problems sustained in a marathon. British Journal of Sports Medicine, 33(1), 22-26.

[11] Ferreira, A. C., Dias, J. M. C., Fernandes, R. de M., Sabino, G. S., dos Anjos, M. T. S., \& Felício, D. C. (2012). Prevalence and associated risks of injury in amateur street runners from Belo Horizonte, MG. Revista Brasileira de Medicina Do Esporte, 18(4), 252-255.

[12] Pope, R. P., Herbert, R. D., Kirwan, J. D., \& Graham, B. J. (2000). A randomized trial of preexercise stretching for prevention of lower-limb injury. Medicine and Science in Sports and Exercise, 32(2), 271-277.

[13] Rauh, M.J., Koepsell, T.D., Rivara, F.P., Rice, S.G., Margherita, A.J. (2007). Quadriceps angle and risk of injury among high school cross-country runners. Journal of Orthopaedics and Sports Physical Therapy, 37: 725-733.

[14] Powers, C. M. (2003). The Influence of Altered Lower-Extremity Kinematics on Patellofemoral Joint Dysfunction: A Theoretical Perspective. Journal of Orthopaedic \& Sports Physical Therapy, 33(11).

[15] Tong, J. W. K., \& Kong, P. W. (2013). Association Between Foot Type and Lower Extremity Injuries: Systematic Literature Review With Meta-analysis. Journal of Orthopaedic \& Sports Physical Therapy, 43(10), 700-714.

[16] Van Mechelen, W. (1992). Running injuries. A review of the epidemiological literature. Sports Medicine (Auckland, N.Z.), 14(5), 320-335.

[17] Macera, C. A. (1992). Lower Extremity Injuries in Runners: Advances in Prediction. Sports Medicine: International Journal of Applied Medicine and Science in Sport and Exercise, 13(1), 50-57. 
[18] Yeung, E. W., \& Yeung, S. S. (2001). A systematic review of interventions to prevent lower limb soft tissue running injuries. British Journal of Sports Medicine.

[19] Jacobs, S. J., \& Berson, B. L. (1986). Injuries to runners: A study of entrants to a 10,000 meter race. The American Journal of Sports Medicine, 14(2), 151-155.

[20] Johnston, C., \& Taunton, J. (2003). Preventing running injuries. Practical approach for family doctors. Canadian Family
Physician, 49, 1101-1109.

[21] Karageanes, S. J. (2004). Principles of Manual Sports Medicine 1st Edition. Lippincott Williams \& Wilkins.

[22] Fredericson, M. (1996). Common injuries in runners. Diagnosis, rehabilitation and prevention. Sports Medicine (Auckland, N.Z.), 21(1), 49-72.

[23] Kvist, M. (1994). Achilles Tendon Injuries in Athletes. Sports Medicine (Auckland, N.Z.), 18(3), 173-201.

(C) The Author(s) 2019. This article is an open access article distributed under the terms and conditions of the Creative Commons Attribution (CC BY) license (http://creativecommons.org/licenses/by/4.0/). 